\title{
Overexpression of CTEN is associated with gefitinib resistance in non-small cell lung cancer
}

\author{
XIANGDONG LU*, YAO ZHANG ${ }^{*}$, YUKAI PAN*, MINMIN CAO, XIE ZHOU and TINGRONG ZHANG \\ Department of Oncology, The Jiangyin Hospital Affiliated to Medical College of Southeast University, \\ Jiangyin, Jiangsu 214400, P.R. China
}

Received May 15, 2020; Accepted October 12, 2020

DOI: $10.3892 / \mathrm{ol} .2020 .12301$

\begin{abstract}
COOH-terminus tensin-like molecule (CTEN) is a member of the tensin family, which is considered to be one of the novel proto-oncogenes involved in tumorigenesis and cancer progression. However, the mechanisms of CTEN in acquired resistance of non-small cell lung cancer (NSCLC) remain relatively unknown. The aim of the present study was to understand the roles of CTEN in acquired gefitinib resistance of NSCLC. The present study investigated the expression level of CTEN using reverse transcription-quantitative polymerase chain reaction and Western blot analysis. Cell Counting kit-8 and colony-formation assays were performed to evaluate the proliferative and colony-formative abilities of PC9 and PC9/GR cells in vitro. Mouse xenograft models were used to assess the growth of PC9/GR cells in vivo. A gefitinib-resistant NSCLC cell line (PC9/GR) was established, and the protein and mRNA expression levels of CTEN were observed to be higher in PC9/GR cells than in PC9 cells. Notably, the sensitivity of PC9/GR cells to gefitinib was observed to be decreased when CTEN was overexpressed, while PC9/GR cells with CTEN-downregulation showed markedly enhanced sensitivity to gefitinib. In vitro proliferation and colony formation assays revealed that increased CTEN markedly promoted the cell proliferative and colony-forming capacities of PC9 and PC9/GR cells, and CTEN-silencing inhibited the cell proliferative and colony-forming abilities of the PC9 and PC9/GR cells. Notably, deficient expression of CTEN notably retarded the growth of PC9/GR xenografts in vivo. In addition, the plasma mRNA expression of CTEN was notably elevated in patients with NSCLC with acquired gefitinib resistance.
\end{abstract}

Correspondence to: Professor Tingrong Zhang, Department of Oncology, The Jiangyin Hospital Affiliated to Medical College of Southeast University, 163 Shoushan Road, Jiangyin, Jiangsu 214400, P.R. China

E-mail: zhangtingrong1967@126.com

*Contributed equally

Key words: $\mathrm{COOH}$-terminus tensin-like molecule, PC9/GR, gefitinib resistance, proliferation, non-small cell lung cancer
Overexpression of CTEN is associated with acquired gefitinib resistance in NSCLC. CTEN may be investigated as a potential therapeutic target for the treatment of patients with NSCLC with acquired gefitinib resistance.

\section{Introduction}

Lung cancer is currently the leading cause of cancer-associated mortalities worldwide. Up to $80 \%$ of patients with lung cancer are diagnosed with non-small cell lung cancer (NSCLC) (1). Over the past decade, epidermal growth factor receptor tyrosine kinase inhibitors (EGFR-TKIs), including gefitinib, have been widely used for the treatment of advanced NSCLC (2). Gefitinib exerts its antitumor effects by abrogating the autophosphorylation of EGFR and subsequently inhibits tumor growth, angiogenesis, vasculogenesis and metastasis (3). Although the efficacy of gefitinib therapy is impressive initially, the majority of patients treated with gefitinib eventually develop gefitinib-resistance, rendering the overall survival of patients with NSCLC unsatisfactory. Therefore, there is an urgent requirement to investigate the underlying molecular mechanisms for advanced NSCLC with acquired gefitinib resistance.

COOH-terminus tensin-like molecule (CTEN, also known as TNS4), a member of the tensin gene family, is involved in cell adhesion, migration and signal transduction (4). Although CTEN is downregulated or absent in advanced prostate cancer, CTEN has been reported to be significantly increased in a variety of malignant tumors, including colorectal (5), breast (6), prostate (7), pancreatic (8), lung cancer (9) and melanoma (10). It has been demonstrated that increased expression of CTEN facilitates cell motility, apoptosis and renal tubulogenesis, suggesting that CTEN may serve important roles in tumorigenesis. In recent years, studies have indicated that CTEN is a promising biomarker and therapeutic target for numerous malignant cancer types (11).

Although CTEN has been demonstrated to be upregulated in NSCLC, the mechanism by which CTEN confers acquired gefitinib resistance in NSCLC remains poorly understood. The present study aimed to investigate the roles of CTEN in acquired gefitinib resistance in NSCLC, which may facilitate the development of a novel therapeutic strategy for the treatment of patients with NSCLC with acquired gefitinib resistance. 


\section{Materials and methods}

Patient samples. Available plasma samples were collected from patients with EGFR-mutant NSCLC $(n=10)$, including 5 patients with an L858R mutation and 5 patients with an exon 19 deletion. The plasma of patients with NSCLC $(n=10)$ was collected prior to and following gefitinib treatment. All patients with NSCLC provided written informed consent and the study was approved by the Ethics Committee of the Jiangyin Hospital Affiliated to Medical College of Southeast University. Detailed clinical information of the enrolled patients are provided in Table SI.

Cell culture and transfection. Human NSCLC PC9 cells were obtained from the Chinese Academy of Sciences Cell Bank of Type Culture Collection and cultured with Dulbecco's modified Eagle's medium (DMEM; Gibco; Thermo Fisher Scientific, Inc.), containing $10 \%$ fetal bovine serum (FBS; Gibco; Thermo Fisher Scientific, Inc.) plus antibiotics $(100 \mathrm{U} / \mathrm{ml}$ penicillin $+100 \mu \mathrm{g} / \mathrm{ml}$ streptomycin) at $37^{\circ} \mathrm{C}$ with $5 \% \mathrm{CO}_{2}$. The gefitinib-resistant NSCLC cell model (PC9/GR) was successfully established by continually exposing PC9 cells to gefitinib for 6 months. For transient transfection, synthesized negative control (NC; GenePharm, Inc.; 5'-UUCUCCGAA CGUGUCACGUTT-3'; 100 nM), CTEN small interfering RNA (siRNA) mimics (GenePharm; 5'-GUGUCUGAUGUC AGCUAUAUG-3'; $100 \mathrm{nM}$ ) and CTEN-overexpressed pCMV plasmid (Clontech Laboratories, Inc.) were transfected using Lipofectamine 2000 reagent (Invitrogen; Thermo Fisher Scientific, Inc.) according to the manufacturer's protocols. A total of $4 \mathrm{~h}$ after transfection, serum-free medium was changed to fresh DMEM medium containing $10 \%$ FBS plus antibiotics at $37^{\circ} \mathrm{C}$ with $5 \% \mathrm{CO}_{2}$.

In vitro analysis of cell proliferation. To assess the cell proliferation ability, a Cell Counting kit-8 (CCK-8) assay (C0038; Beyotime Institute of Biotechnology) was performed in vitro. PC9 and PC9/GR cells were implanted into 96-well plates $\left(3 \times 10^{3}\right.$ cells/well). At the times indicated (days 0, 1, 2 and 3), absorption at $450 \mathrm{~nm}$ (OD450) was determined following incubation with $10 \mu \mathrm{l}$ CCK- 8 reagent by spectrophotometry (Bio Rad Laboratories, Inc.).

In vitro colony formation assay. In vitro colony-formation assay was performed to investigate the in vitro colony-formation ability. PC9 and PC9/GR cells were seeded onto a 6-well plate (1,000 cells/well), and cultured with DMEM medium (Gibco; Thermo Fisher Scientific, Inc.) containing 10\% FBS (Gibco; Thermo Fisher Scientific, Inc.) for two weeks at $37^{\circ} \mathrm{C}$ with $5 \% \mathrm{CO}_{2}$. Cells were fixed with $100 \%$ methanol for $20 \mathrm{~min}$ at room temperature and stained with $0.25 \%$ crystal violet (dissolved in 20\% methanol) for $30 \mathrm{~min}$ at room temperature. The images of colonies were captured with a GT-X700 scanner (Epson Corp.).

RNA isolation and reverse transcription-quantitative polymerase chain reaction $(R T-q P C R)$. Total RNA was extracted using the standard TRIzol method (Thermo Fisher Scientific, Inc.) and the cDNA synthesis was performed using the HiScript Q RT SuperMix for qPCR (+gDNA wiper; R123-01,
Vazyme Biotech Co., Ltd.) under the following conditions: $42^{\circ} \mathrm{C}$ for $2 \mathrm{~min}, 50^{\circ} \mathrm{C}$ for $15 \mathrm{~min}$ and $85^{\circ} \mathrm{C}$ for $2 \mathrm{~min}$. qPCR was performed using AceQ Universal SYBR qPCR Master Mix (Q511-02; Vazyme Biotech Co., Ltd.) using the StepOnePlus Real-Time PCR System (Bio-Rad Laboratories, Inc.). All reactions were run in duplicate and $10 \mu \mathrm{l}$ aliquots of the reaction mixture were pipetted into each well of a 96-well PCR plate (Bio-Rad Laboratories, Inc.). The thermocycling conditions were as follows: $95^{\circ} \mathrm{C}$ for $10 \mathrm{~min}$, then 40 cycles of $95^{\circ} \mathrm{C}$ for $30 \mathrm{sec}, 60^{\circ} \mathrm{C}$ for $30 \mathrm{sec}$ and $72^{\circ} \mathrm{C}$ for $60 \mathrm{sec}$. Relative mRNA amounts of target genes were calculated following normalization to an endogenous reference gene (GAPDH) according to the arithmetic formula $2^{-\Delta \Delta \mathrm{Cq}}(12)$. The real-time PCR primer sequences were: CTEN forward, 5'-ACTGATGTCCAGAGG AAGGTG-3' and reverse, 5'-ATGTCATACTCCGCAAAG AGG-3'; GAPDH forward, 5'-GGTCTCCTCTGACTTCAA CA-3' and reverse, 5'-AGCCAAATTCGTTGTCATAC-3'.

Protein extraction and Western blot analysis. Cells were washed twice with 1X PBS, and lysed with RIPA buffer (25 mM Tris, $150 \mathrm{mM} \mathrm{NaCl,} \mathrm{0.1 \%} \mathrm{SDS,} \mathrm{1 \%} \mathrm{NP40,} 2 \mathrm{mM}$ EDTA and 1\% Triton X-100; pH 7.4). Protein concentration was determined by the bicinchoninic acid (BCA) protein assay kit (Pierce; Thermo Fisher Scientific Inc.). Cell lysates were mixed with $5 \mathrm{X}$ loading buffer and denatured at $100^{\circ} \mathrm{C}$ for $10 \mathrm{~min}$. Next, protein samples ( $40 \mu \mathrm{g}$ per lane) were subjected to $10 \%$ SDS-PAGE and transferred onto a $45-\mu \mathrm{m}$ polyvinylidene fluoride membrane (EMD Millipore) using a semi-dry electrophoresis transfer apparatus (Bio-Rad Laboratories, Inc.). Next, the membrane was blocked with PBST (1X PBS $+0.1 \%$ Tween-20) containing $5 \%(\mathrm{w} / \mathrm{v})$ skimmed milk for $1 \mathrm{~h}$ at room temperature. The blots were then incubated with anti-CTEN (cat. no. ab192247; dilution, 1:1,000; Abcam) and anti-b-actin (cat. no. ab8227; dilution, 1:3,000; Abcam) antibodies overnight at $4^{\circ} \mathrm{C}$. Following washing with PBST and incubation with a horseradish peroxidase-conjugated secondary rabbit antibody for $1 \mathrm{~h}$ (cat. no. sc-2357; dilution, 1:3,000; Santa Cruz Biotechnology) at room temperature, the protein bands were visualized using an ECL detection kit (Thermo Fisher Scientific, Inc.).

In vivo xenograft assays. All animal experimental protocols were approved by the Animal Research Ethics Committee of Jiangyin Hospital affiliated to Medical College of Southeast University. BALB/c nude mice (6-week-old, female, $n=10$ ) were purchased from the Animal Laboratory Center of Nanfang Medical University (Guangdong, China). All mice were housed under a fixed light-dark cycle (12 h of light and $12 \mathrm{~h}$ of darkness) in a laminar flow room at constant temperature and humidity, with ad libitum access to sterilized food and water. CTEN-short hairpin RNA (shRNA) and negative control shRNA (shRNA-NC) were designed and synthesized, prior to being inserted into the lentiviral vector pLKO.1-TRC (1st generation; cat. no. 10879; Addgene Inc.). Control and pLKO.1-CTEN-shRNA were co-transfected into HEK-293T cells together with lentivirus packaging plasmids (PMD2G, psPAX2) (pLKO.1-CTEN-shRNA:PMD2G:psPAX2=1:1:1) to prepare the lentiviruses using Lipofectamine 2000 (Thermo Fisher Scientific, Inc.). Subsequently, PC9/GR cells $\left(2 \times 10^{6}\right.$ cells in PBS/100 $\left.\mu \mathrm{l}\right)$ were infected with shRNA-NC or 

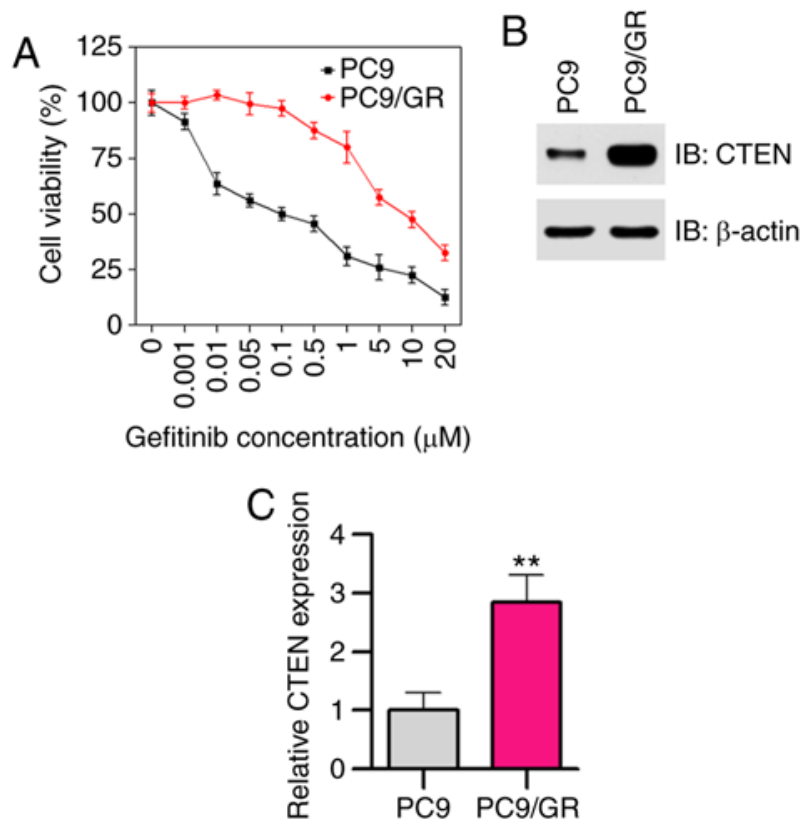

Figure 1. CTEN is upregulated in human non-small cell lung cancer gefitinib-resistant cells. (A) The sensitivities of PC9 and PC9/GR cells to different concentrations of gefitinib $(0.001-20 \mu \mathrm{M})$ were evaluated using Cell Counting Kit-8 assays. (B) Western blot analysis of CTEN protein expression in PC9 and PC9/GR cells. $\beta$-actin was used as endogenous loading control. (C) Reverse transcription-quantitative polymerase chain reaction analysis of CTEN expression levels in PC9 and PC9/GR cells. ${ }^{* * *} \mathrm{P}<0.01$. Experiments were performed in triplicates. Data are presented as the mean \pm standard deviation. CTEN, $\mathrm{COOH}$-terminus tensin-like molecule PC9/GR, gefitinib-resistant PC9 cells.

CTEN shRNA-viruses and selected using puromycin, prior to being injected subcutaneously into the flanks of BALB/c nude mice ( 5 mice per group). Mice were euthanized when tumors ( $\mathrm{shRNA-NC)}$ reached $>1 \mathrm{~cm}$ in diameter. All mice were weighed and the tumor size was measured every 3 days using an electronic-caliper. Tumor volume was calculated using the equation: $\mathrm{V}=\left(\mathrm{LxW}^{2}\right) / 2$, where $\mathrm{V}$ represents the tumor volume, $\mathrm{L}$ represents the length and $\mathrm{W}$ represents the width. Mice were sacrificed at day 21 and tumors were surgically excised from the mice.

Statistical analysis. The results were performed in triplicate, and the results are expressed as the mean \pm standard deviation. Student's t-test was performed to analyze the data. Furthermore, paired t-tests were performed to compare the expression of CTEN mRNA in plasma samples collected prior to and following gefitinib treatment. $\mathrm{P}<0.05$ was considered to indicate a statistically significant difference.

\section{Results}

CTEN is upregulated in gefitinib-resistant NSCLC cells. In the present study, a gefitinib-resistant cell line, PC9/GR, was established by increasing the concentrations of gefitinib in a stepwise manner in vitro. Half inhibition concentration (IC50) values of gefitinib in PC9 and PC9/GR cells treated with different concentrations of gefitinib ranging between 0.001 and $20 \mu \mathrm{M}$ for $48 \mathrm{~h}$ were evaluated using CCK-8 assays. As expected, PC9/GR cells were found to be resistant to gefitinib
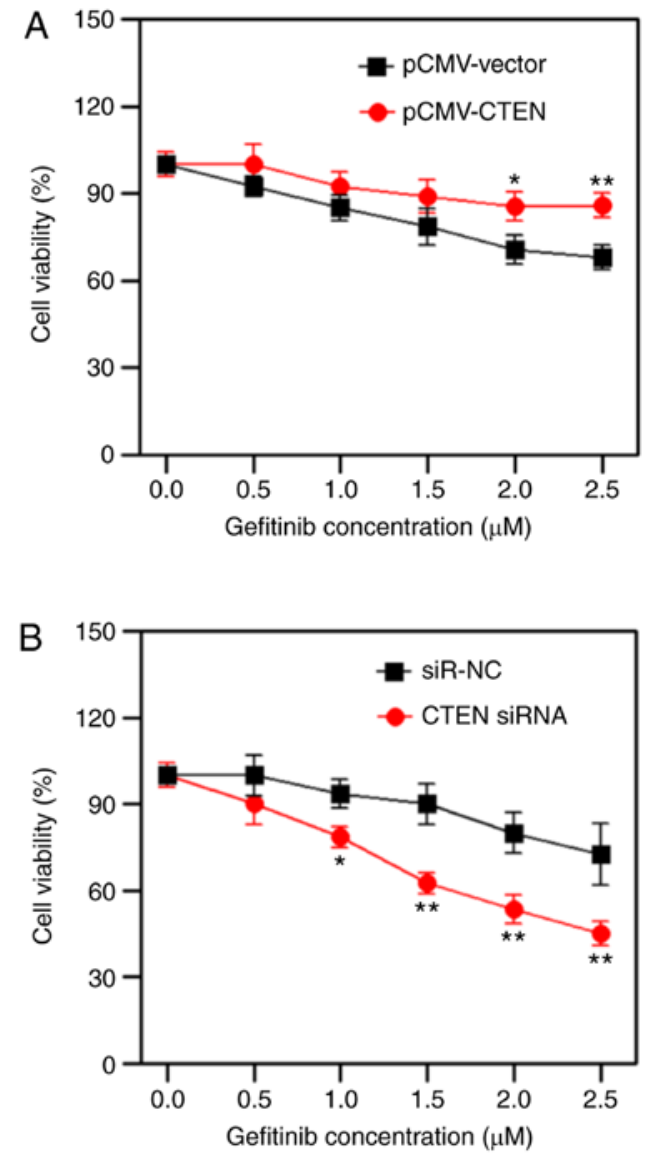

Figure 2. CTEN attenuates the sensitivity of PC9/GR cells to gefitinib. (A) The sensitivities of pCMV-vector- and pCMV-CTEN-transfected PC9/GR cells to gefitinib were evaluated by CCK- 8 assays. ${ }^{*} \mathrm{P}<0.05,{ }^{* *} \mathrm{P}<0.01$. Experiments were performed in triplicate. (B) The sensitivities of siR-NCand CTEN siRNA-transfected PC9/GR cells to gefitinib were evaluated by CCK- 8 assays. Experiments were performed in triplicate. Data are presented as the mean \pm standard deviation. CTEN, COOH-terminus tensin-like molecule; PC9/GR, gefitinib-resistant PC9 cells; CCK-8, Cell Counting Kit-8; siR/siRNA, small interfering RNA; NC, negative control.

$\left(\mathrm{IC}_{50}=8.6 \mu \mathrm{M}\right)$, and PC9 cells were found to be sensitive to gefitinib (IC50=0.10 $\mu \mathrm{M}$; Fig. 1A). To examine the possible role of CTEN in acquired resistance to gefitinib, the expression of CTEN in gefitinib-sensitive PC9 and gefitinib-resistant PC9/GR cells was investigated. As shown in Fig. 1B and C, the protein and mRNA expression levels of CTEN were higher in the PC9/GR cells compared with those in the PC9 cells $(\mathrm{P}<0.01)$, suggesting that $\mathrm{CTEN}$ may serve potential roles of in acquired gefitinib resistance.

CTEN attenuates the sensitivity of PC9/GR cells to gefitinib. To elucidate the mechanistic and functional aspects of CTEN in modulating the sensitivity of NSCLC cells to gefinib in vitro, the expression of CTEN in PC9/GR cells was upregulated by transfection using the pCMV-CTEN plasmid, following which the gefitinib-sensitivity of transfected PC9/GR cells was determined using CCK-8 assays. Notably, as shown in Fig. 2A, the sensitivity of PC9/GR cells to gefitinib was decreased when CTEN was overexpressed $(\mathrm{P}<0.05, \mathrm{P}<0.01)$. Next, the expression of CTEN in PC9/GR cells was downregulated by siRNA transfection, and the sensitivity of transfected PC9/GR cells to gefitinib was investigated using CCK- 8 assays. As 

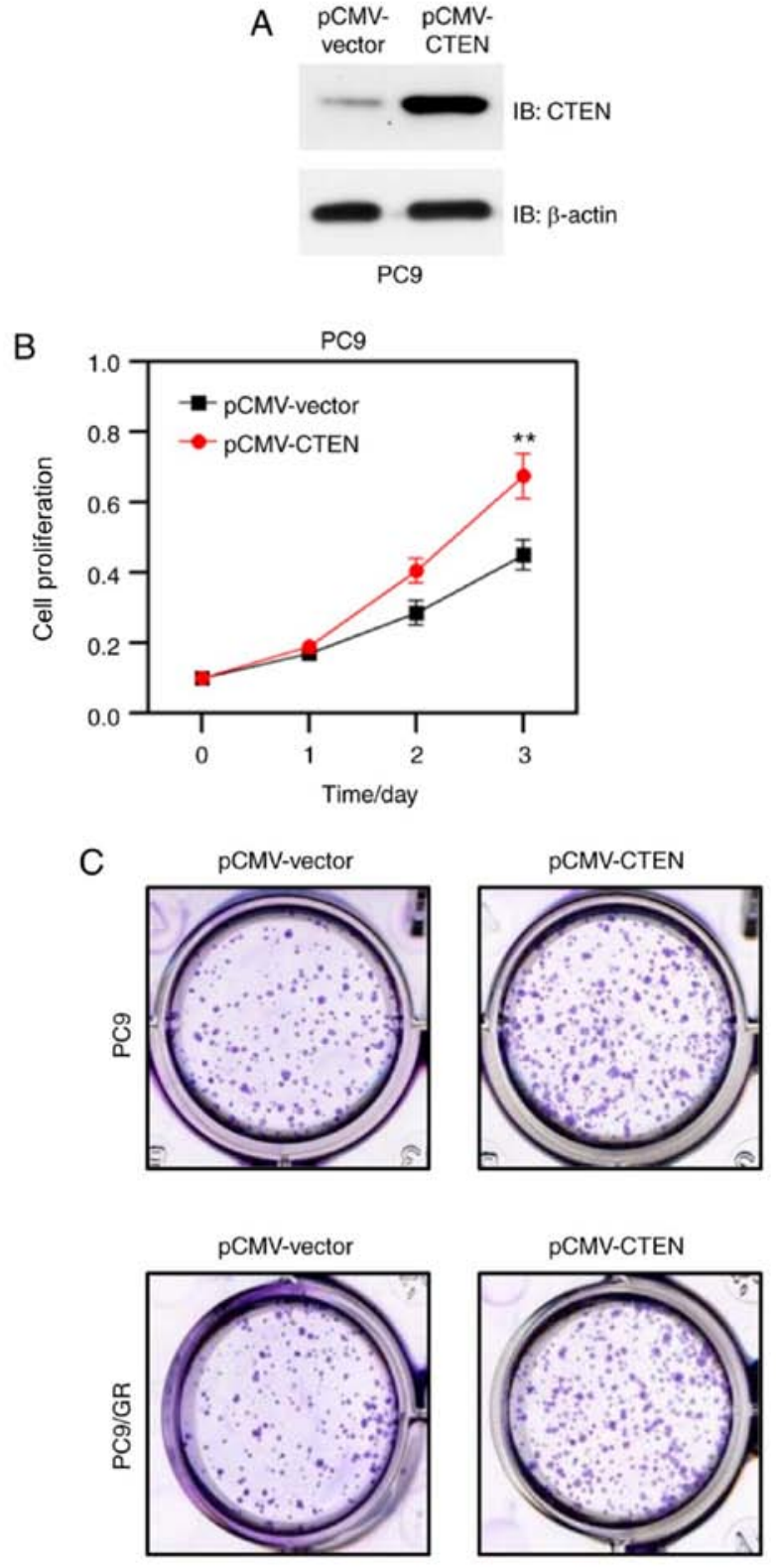

Figure 3. Overexpression of CTEN promotes the proliferation of NSCLC cells. (A) Western blot analysis of CTEN protein expression in pCMV-vector- and pCMV-CTEN-transfected PC9 and PC9/GR cells. $\beta$-actin was used as endogenous loading control. (B) The proliferative abilities of pCMV-vector- and pCMV-CTEN-transfected PC9 and PC9/GR cells were evaluated by Cell Counting Kit- 8 assays. ${ }^{* * *} \mathrm{P}<0.01$. Experiments were performed in triplicate. (C) The colony-forming abilities of pCMV-vector- and pCMV-CTEN-transfected PC9 and PC9/GR cells were evaluated by plate colony-formation assays. ${ }^{* *} \mathrm{P}<0.01$ Experiments were performed in triplicate. Data are presented as the mean \pm standard deviation. CTEN, COOH-terminus tensin-like molecule; PC9/GR, gefitinib-resistant PC9 cells.

expected, PC9/GR cells with CTEN downregulation showed markedly enhanced sensitivity to gefitinib when the cells were treated with different concentrations of gefitinib (Fig. 2B; $\mathrm{P}<0.05, \mathrm{P}<0.01)$. Taken together, these results demonstrated that CTEN-overexpression may attenuate the sensitivity of PC9/GR cells to gefitinib.

Overexpression of CTEN promotes the proliferation of NSCLC cells. The effect of CTEN-overexpression on the proliferative ability of gefitinib-sensitive (PC9) and gefitinib-resistant (PC9/GR) cells was investigated. Western blot analysis revealed that CTEN was markedly upregulated in PC9 and PC9/GR cells transfected with the pCMV-CTEN
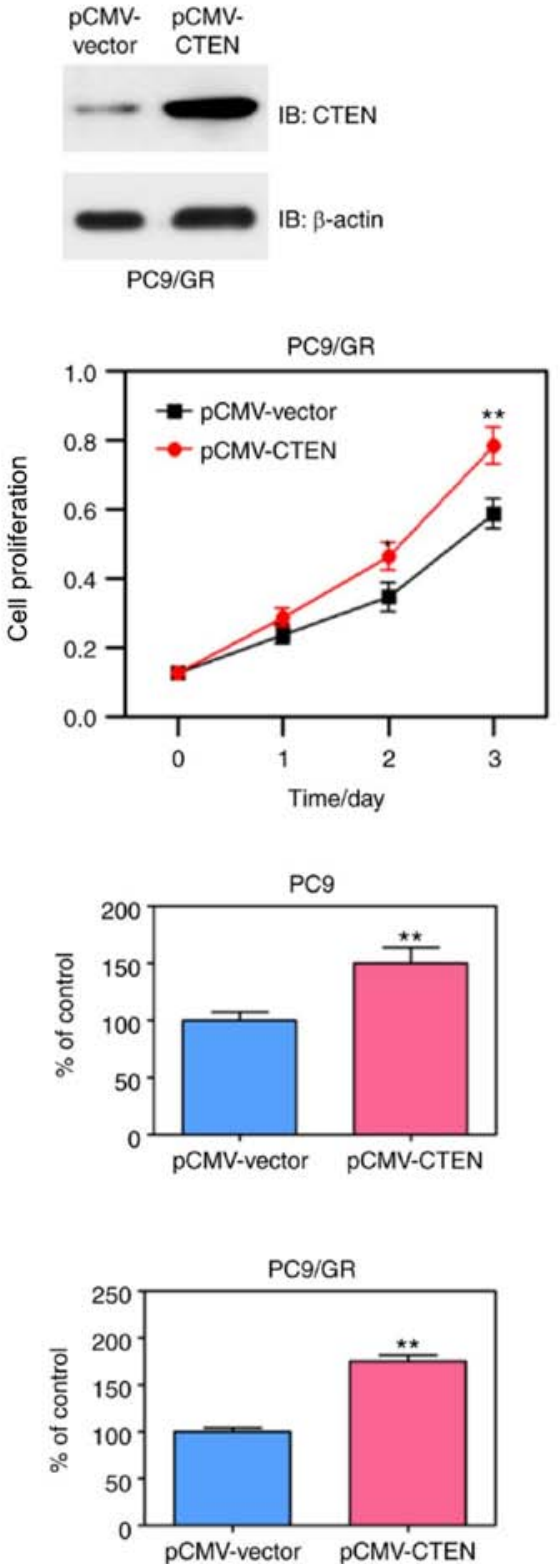

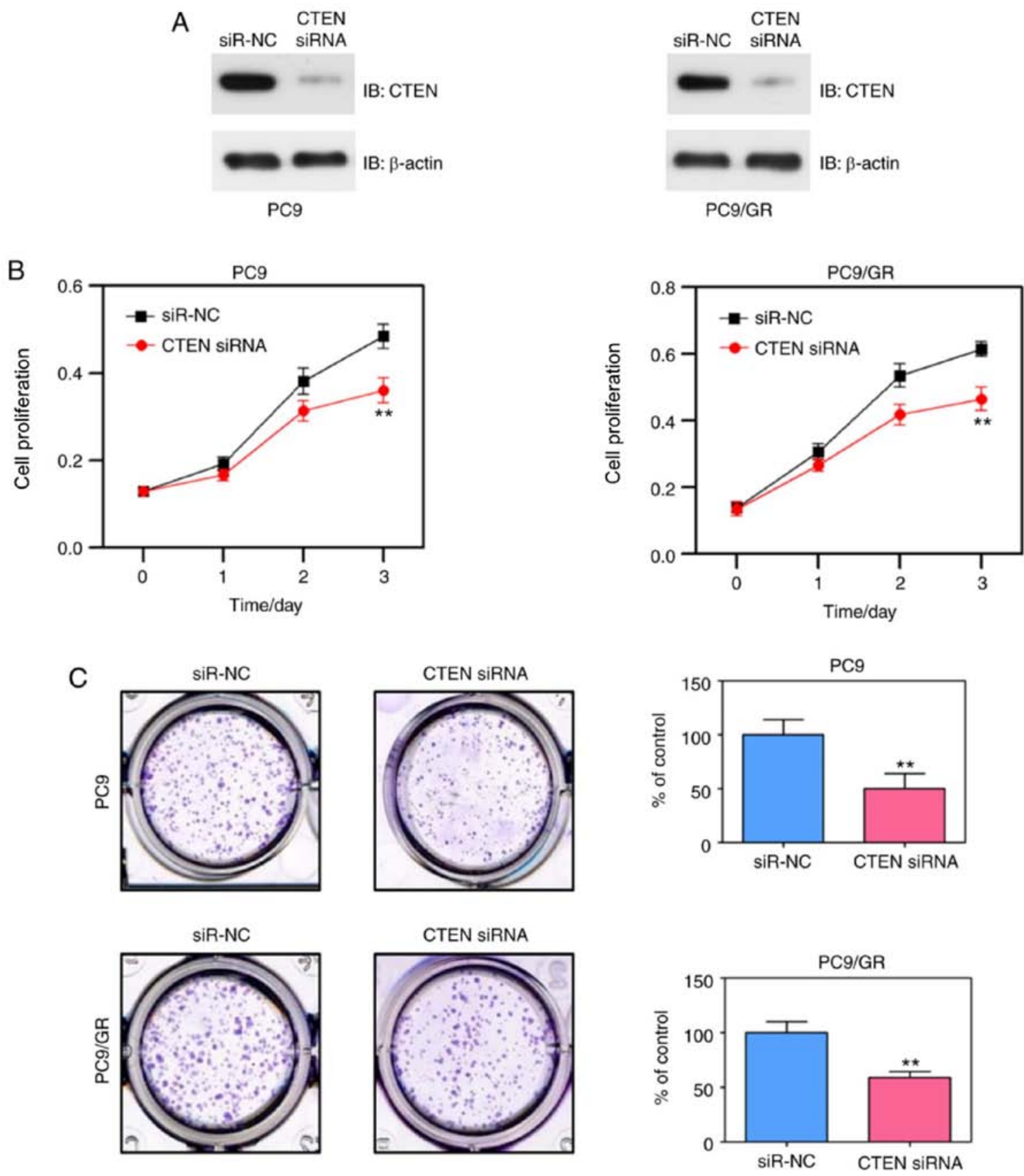

Figure 4. Knockdown of CTEN inhibits the proliferation of non-small cell lung cancer cells. (A) Western blot analysis of CTEN protein expression in siR-NC- and CTEN siRNA-transfected PC9 and PC9/GR cells. $\beta$-actin was used as endogenous loading control. (B) The proliferative abilities of siR-NCand CTEN siRNA-transfected PC9 and PC9/GR cells were evaluated by Cell Counting Kit- 8 assays. ${ }^{* *} \mathrm{P}<0.01$. Experiments were performed in triplicate. (C) The colony-forming abilities of siR-NC- and CTEN siRNA-transfected PC9 and PC9/GR cells were evaluated by plate colony-formation assays. ** P $<0.01$. Experiments were performed in triplicate. Data are presented as the mean \pm standard deviation. CTEN, COOH-terminus tensin-like molecule; siR/siRNA, small interfering RNA; NC, negative control; PC9/GR, gefitinib-resistant PC9 cells.

transfected with CTEN-siRNA. As shown in Fig. 4A, cells transfected with CTEN-siRNA resulted in a significant loss of CTEN expression compared with that in cells transfected with NC-siRNA. Next, the effect of CTEN-knockdown on cell proliferation was investigated using CCK-8 assays. As shown in Fig. 4B, the proliferative capabilities of gefitinib-sensitive and gefitinib-resistant NSCLC cells were observed to be significantly decreased $(\mathrm{P}<0.01)$ when the expression of CTEN was downregulated. Consistently, siRNA-mediated knockdown of CTEN significantly suppressed the colony-forming abilities of PC9 and PC9/GR cells (Fig. 4C; P<0.01).
Depletion of CTEN suppresses the growth of PC9/GR xenografts in vivo. The aforementioned phenotypic analysis demonstrated that CTEN-knockdown significantly decreased the proliferative and colony-formative capabilities of PC9/GR cells in vitro. To further confirm the oncogenetic roles of CTEN in NSCLC, the effects of lentivirus-mediated CTEN depletion on the growth of PC9/GR cells in vivo were investigated. Notably, as shown in Fig. 5A and B $(\mathrm{P}<0.01)$, CTEN depletion suppressed the growth of PC9/GR xenografts in vivo. Furthermore, there was no significant difference observed between the body weight of shRNA-NC- and 

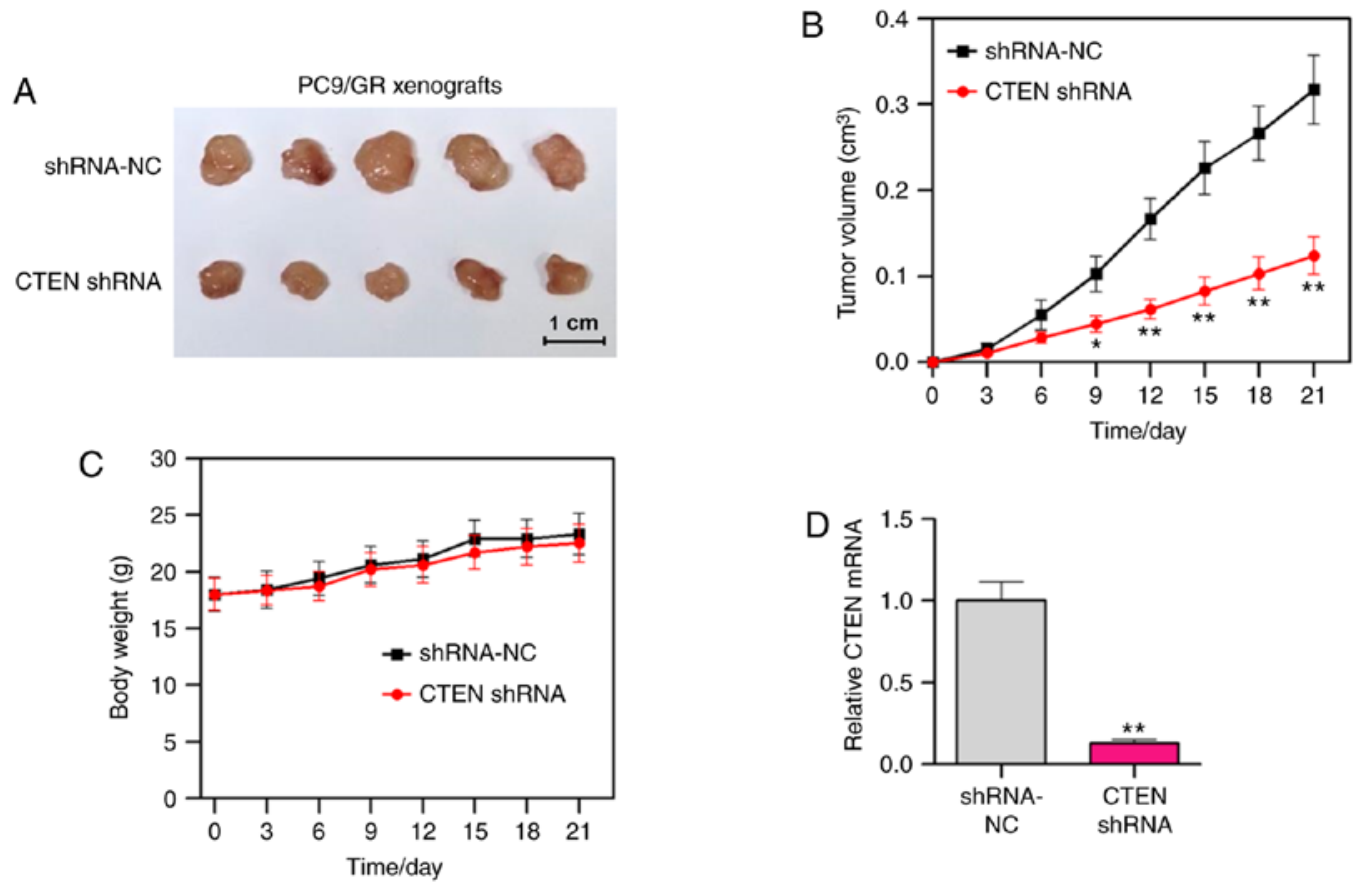

Figure 5. Depletion of CTEN suppresses the growth of PC9/GR xenografts in vivo. (A) Representative image shows subcutaneous xenografts excised from mice injected with shRNA-NC- or CTEN shRNA-treated PC9/GR cells. (B) Tumor growth curves of shRNA-NC- or CTEN shRNA-treated PC9/GR xenografts. (C) Body weight of mice injected with shRNA-NC- or CTEN shRNA-treated PC9/GR cells. (D) Relative mRNA expression of CTEN was validated by reverse transcription-quantitative polymerase chain reaction in shRNA-NC- or CTEN shRNA-treated PC9/GR xenografts. Data are presented as the mean \pm standard deviation. ${ }^{*} \mathrm{P}<0.05,{ }^{* *} \mathrm{P}<0.01$. CTEN, COOH-terminus tensin-like molecule; PC9/GR, gefitinib-resistant PC9 cells; shRNA, short hairpin RNA; NC, negative control.

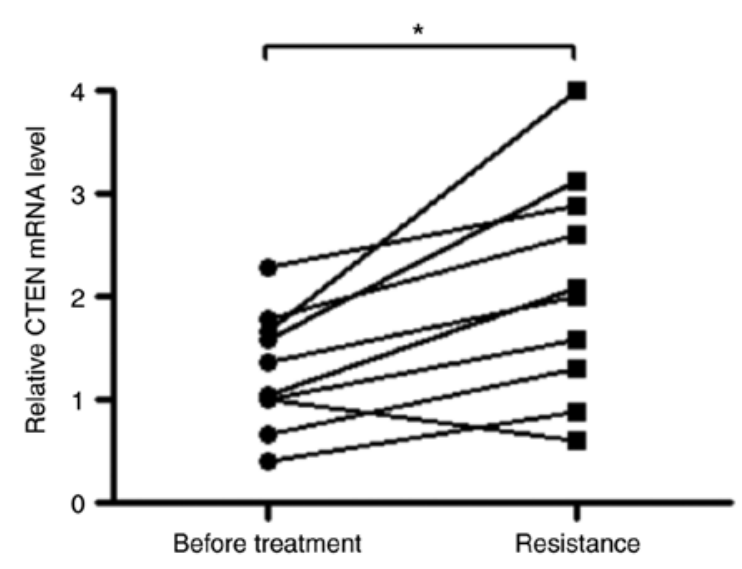

Figure 6. CTEN was upregulated in patients with NSCLC with acquired resistance to gefitinib. Reverse transcription-quantitative polymerase chain reaction analysis of CTEN mRNA in the plasma samples from 10 patients with NSCLC collected prior to and following gefitinib treatment (resistance). ${ }^{*} \mathrm{P}<0.05$. CTEN, COOH-terminus tensin-like molecule; NSCLC, non-small cell lung cancer.

CTEN shRNA-treated mice (Fig. 5C). Based on the results of RT-qPCR, the mRNA expression CTEN in CTEN-depleted PC9/GR xenografts was significantly lower than that in the control PC9/GR xenografts (Fig. 5D; P<0.01). Taken together, these results further suggested that CTEN is required for the growth of PC9/GR xenografts, emphasizing the important role of CTEN in promoting NSCLC progression.

CTEN is upregulated in patients with NSCLC with acquired resistance to gefitinib. To determine the expression levels of CTEN in patients with NSCLC treated with EGFR-TKI, plasma samples from 10 patients with NSCLC were collected prior to and following gefitinib treatment, following which total RNA was isolated for CTEN expression evaluation. As shown in Fig. 6, the mRNA expression of CTEN was significantly increased in patients with NSCLC with acquired gefitinib resistance $(\mathrm{P}<0.05)$.

\section{Discussion}

Somatic mutations of EGFR may facilitate the development of effective EGFR-TKIs for successfully treating patients with NSCLC. However, chemoresistance development is inevitable, and the majority of patients with NSCLC experience highly malignant and aggressive relapses. Therefore, there is an urgent requirement to identify the prognostic indicators and to elucidate the underlying molecular mechanisms for advanced NSCLC with acquired gefitinib resistance. The results of the present study demonstrated that upregulation of CTEN may promote cell proliferation and colony formation of gefitinib-resistant NSCLC cells, suggesting that CTEN may contribute toward gefitinib resistance in NSCLC.

Lo et al (11) have suggested that CTEN expression is frequently increased in a variety of malignant tumors, including colorectal, gastric, pancreatic and lung cancer (11). Thorpe et al (13) demonstrated that CTEN may facilitate epithelial-mesenchymal transition (EMT) processes by preventing the degradation of Snail protein in colorectal cancer. Asiri et al (14) demonstrated that CTEN is a positive modulator of Src, and that CTEN may promote EMT and metastasis via post-transcriptional stabilization of Src protein 
in colorectal cancer. Notably, Hong et al (15) revealed that a proportion of nuclear-localized CTEN contributes toward cell proliferation in cancer cells. Wu et al (16) demonstrated that knockdown of CTEN inhibits prostate cancer cell proliferation and results in $\mathrm{G}_{1} / \mathrm{S}$ cell cycle arrest, suggesting that inhibition of CTEN expression is required for luminal differentiation and acinar formation. Consistently, our previous study demonstrated that CTEN may activate the expression of TGF- $\beta 1$, which promotes EMT and metastasis in NSCLC cells, thereby strongly supporting the role of CTEN as an oncoprotein in NSCLC (17).

Over the last decade, a number of potential mechanisms that may lead to acquired resistance to gefitinib in NSCLC have been proposed. Using a genome-wide RNAi screening approach, Cho et al (18) examined a synthetic lethality with gefitinib in EGFR-mutated TKI-resistant NSCLC cells, and identified RNF25 as an important modulator that may enhance the acquired gefitinib resistance. Furthermore, they revealed that RNF25 may serve crucial roles in the contribution of acquired gefitinib resistance by facilitating the cross-talk between NF- $\kappa \mathrm{B}$ and ERK signaling pathways in NSCLC (18). Li et al (19) suggested that downregulation of RHPN1-AS1 IncRNA contributes toward acquired gefitinib resistance by targeting the miR-299-3p/TNFSF12 pathway in NSCLC. Lu et al (20) reported that hypoxia is a driving force for acquired gefitinib resistance through the regulation of the EMT process, and epigenetic regulators, including LSD1 and PLU-1, thereby serving crucial roles in hypoxia-induced gefitinib resistance. Li et al (21) investigated the role of DEAD-Box Helicase 17 (DDX17) in acquired gefitinib resistance and observed that exportin/importin-dependent nucleocytoplasmic shuttling of DDX17 serves important roles in acquired gefitinib resistance through the activation of $\beta$-catenin in NSCLC. Overexpression of peptidylarginine deiminase IV (PAD4) has been demonstrated to decrease the activity of EMT by inhibiting the expression of ETS-domain containing protein (Elk1), which then suppresses the resistance of NSCLC cell lines to gefitinib (22). Feng et al (23) indicated that Krüppel-like factor 4 (KLF4) is an important contributing factor for gefitinib resistance in c-Met-overexpressed-NSCLC cells by repressing the expression of apoptosis-related proteins. In addition, microRNAs have been recently reported to be widely involved in acquired gefitinib resistance in NSCLC $(24,25)$. In conclusion, the mechanisms of gefitinib resistance remain unclear. Further studies are urgently required to elucidate the underlying mechanisms of gefitinib resistance in NSCLC.

Identification of potential factors that regulate the resistance of NSCLC cells to EGFR-TKIs, including gefitinib may provide more precise information that facilitates effective therapy. The present study identified that CTEN is upregulated in gefitinib-resistant NSCLC cells, and that CTEN may lower the sensitivity of gefitinib-resistant NSCLC cells to gefitinib. Subsequently, it was observed that CTEN exerts a promoting effect on the growth of gefitinib-resistant NSCLC cells in vitro or in vivo. In addition, the mRNA expression of CTEN is significantly increased in patients with NSCLC with resistance to gefitinib, suggesting that CTEN may contribute toward gefitinib resistance in NSCLC. In conclusion, CTEN may be a potential therapeutic target for the treatment of patients with NSCLC with acquired gefitinib resistance. However, the present study is confined to a small number of enrolled patients with NSCLC. Therefore, prospective studies with a larger number of NSCLC patients are required to validate the findings of the present study.

\section{Acknowledgements}

Not applicable.

\section{Funding}

The present study was supported by the Wuxi Science and Technology Project (2018) and the Youth Fund of Wuxi Health Committee (grant no. 201904).

\section{Availability of data and materials}

The datasets used and/or analyzed during the current study are available from the corresponding author on reasonable request.

\section{Authors' contributions}

$\mathrm{XL}, \mathrm{YZ}$ and YP conducted the experiments, analyzed and interpreted the data. MC and XZ aided in collecting plasma samples from patients with NSCLC. TZ conceived this study and wrote the manuscript. All authors read and approved the final manuscript.

\section{Ethics statement and consent to participate}

All patients provided written informed consent and the present study was approved by the Ethics Committee of the Jiangyin Hospital affiliated to Medical College of Southeast University. All animal experimental protocols were approved by the Animal Research Ethics Committee of Jiangyin Hospital affiliated to Medical College of Southeast University.

\section{Patient consent for publication}

All patients provided written informed consent for publication.

\section{Competing interests}

The authors declare that they have no competing interests.

\section{References}

1. Siegel R, Naishadham D and Jemal A: Cancer statistics, 2013. CA Cancer J Clin 63: 11-30, 2013.

2. Ansari J, Palmer DH, Rea DW and Hussain SA: Role of tyrosine kinase inhibitors in lung cancer. Anticancer Agents Med Chem 9: 569-575, 2009.

3. Muhsin M, Graham J and Kirkpatrick P: Gefitinib. Nat Rev Drug Discov 2: 515-516, 2003.

4. Lo HS and Lo TB: Cten, a COOH-terminal tensin-like protein with prostate restricted expression, is down-regulated in prostate cancer. Cancer Res 62: 4217-4221, 2002.

5. Albasri A, Seth R, Jackson D, Benhasouna A, Crook S, Nateri AS, Chapman R and Ilyas M: C-Terminal Tensin-like (CTEN) is an oncogene which alters cell motility possibly through repression of E-cadherin in colorectal cancer. J Pathol 218: 57-65, 2009.

6. Albasri A, Aleskandarany M, Benhasouna A, Powe DG, Ellis IO, Ilyas M and Green AR: CTEN (C-terminal tensin-like), a novel oncogene overexpressed in invasive breast carcinoma of poor prognosis. Breast Cancer Res Treat 126: 47-54, 2011. 
7. Li YQ, Mizokami A, Izumi K, Narimoto K, Shima T, Zhang J, Dai J, Keller ET and Namiki M: CTEN/tensin 4 expression induces sensitivity to paclitaxel in prostate cancer. Prostate 70 : $48-60,2010$

8. Al-Ghamdi S, Cachat J, Albasri A, Ahmed M, Jackson D, Zaitoun A, Guppy N, Otto WR, Alison MR, Kindle KB and Ilyas M: C-Terminal tensin-like gene functions as an oncogene and promotes cell motility in pancreatic cancer. Pancreas 42 : $135-140,2013$

9. Sasaki H, Moriyama S, Mizuno K, Yukiue H, Konishi A, Yano M, Kaji M, Fukai I, Kiriyama M, Yamakawa Y and Fujii Y: Cten mRNA expression was correlated with tumor progression in lung cancers. Lung Cancer 40: 151-155, 2003.

10. Sjoestroem C, Khosravi S, Zhang G, Martinka M and Li G: $\mathrm{C}$-Terminal tensin-like protein is a novel prognostic marker for primary melanoma patients. PLoS One 8: e80492, 2013.

11. Lo SH: C-Terminal tensin-like (CTEN): A promising biomarker and target for cancer. Int J Biochem Cell Biol 51: 150-154, 2014.

12. Livak KJ and Schmittgen TD: Analysis of relative gene expression data using real-time quantitative PCR and the 2(-Delta Delta C(T)) method. Methods 25: 402-408, 2001.

13. Thorpe H, Asiri A, Akhlaq M and Ilyas M: Cten promotes epithelial-mesenchymal transition through post-transcriptional stabilization of snail. Mol Carcinog 56: 2601-2609, 2017.

14. Asiri A, Toss MS, Raposo TP, Akhlaq M, Thorpe H, Alfahed A, Asiri A and Ilyas M: Cten promotes epithelial-mesenchymal transition (EMT) in colorectal cancer through stabilisation of src. Pathol Int 69: 381-391, 2019.

15. Hong SY, Shih YP, Lo A and Lo SH: Identification of subcellular targeting sequences of Cten reveals its role in cell proliferation. Biochim Biophys Acta Mol Cell Res 1866: 450-458, 2019.

16. Wu WM and Liao YC: Downregulation of C-terminal tensin-like protein (CTEN) suppresses prostate cell proliferation and contributes to acinar morphogenesis. Int J Mol Sci 19: 3190, 2018.

17. Lu X, Gao J, Zhang Y,Zhao T, Cai H and Zhang T: CTEN induces epithelial-mesenchymal transition (EMT) and metastasis in non small cell lung cancer cells. PLoS One 13: e0198823, 2018.
18. Cho JH, You YM, Yeom YI, Lee DC, Kim BK, Won M, Cho BC, Kang M, Park S, Yang SJ, et al: RNF25 promotes gefitinib resistance in EGFR-mutant NSCLC cells by inducing NF- $\kappa B$-mediated ERK reactivation. Cell Death Dis 9: 587, 2018.

19. Li X, Xin Z, Chunlu Y, Su C, Qiming S and Shun X: The lncRNA RHPN1-AS1 downregulation promotes gefitinib resistance by targeting miR-299-3p/TNFSF12 pathway in NSCLC. Cell Cycle 17: 1772-1783, 2018.

20. Lu Y, Liu YF, Sebastian O and Glazer PM: Hypoxia promotes resistance to EGFR inhibition in NSCLC cells via the histone demethylases, LSD1 and PLU-1. Mol Cancer Res 16: 1458-1469, 2018.

21. Li K, Mo C, Gong D, Chen Y, Huang Z, Li Y, Zhang J, Huang L, Li Y, Fuller-Pace FV, et al: DDX17 nucleocytoplasmic shuttling promotes acquired gefitinib resistance in non-small cell lung cancer cells via activation of $\beta$-catenin. Cancer Lett 400: 194-202, 2017.

22. Duan Q, Pang C, Chang N, Zhang J and Liu W: Overexpression of PAD4 suppresses drug resistance of NSCLC cell lines to gefitinib through inhibiting Elk1-mediated epithelial-mesenchymal transition. Oncol Rep 36: 551-558, 2016.

23. Feng W, Xie Q, Liu S, Ji Y and Jin L: KLF4 promotes c-met amplification-mediated gefitinib resistance in NSCLC. Cancer Sci 109: 1775-1786, 2018.

24. Jiao D, Chen J, Li Y, Tang X, Wang J, Xu W, Song J, Li Y, Tao H and Chen Q: miR-1-3p and miR-206 sensitizes HGF-induced gefitinib-resistant human lung cancer cells through inhibition of c-Met signalling and EMT. J Cell Mol Med 22: 3526-3536, 2018.

25. Cao X, Lai S, Hu F, Li G, Wang G, Luo X, Fu X and Hu J: miR-19a contributes to gefitinib resistance and epithelial mesenchymal transition in non-small cell lung cancer cells by targeting c-Met. Sci Rep 7: 2939, 2017.

This work is licensed under a Creative Commons Attribution-NonCommercial-NoDerivatives 4.0 International (CC BY-NC-ND 4.0) License. 